\title{
The HS-SAS and GSH-SAS Distribution as Model for Unconditional and Conditional Return Distributions
}

\author{
Matthias Fischer and Klaus Herrmann \\ University of Erlangen-Nürnberg, Germany
}

\begin{abstract}
We introduce two new skewed and leptokurtic distributions derived from the hyperbolic secant distribution and from Vaughan (2002)'s generalized hyperbolic distribution by use of the sinh-arcsinh transformation introduced in Jones and Pewsey (2009). Properties of these new distribution are given. Their flexibility for modeling financial return data is comparable to that of their most advanced peers. Contrary to the latter for both distributions a closed-form solution for the density, cumulative distribution and quantile function can be given.
\end{abstract}

Zusammenfassung: Im Rahmen der Arbeit werden zwei neue, flexible Verteilungsfamilien eingeführt, die sich von der hyperbolischen Sekantverteilung bzw. von Vaughan (2002)'s verallgemeinert hyperbolischer Sekantverteilung durch Anwendung der sogenannten sinh-arcsinh Transformation ableiten lassen. Beide Verteilungsklassen erlauben für die Dichte-, die Verteilungs- sowie die Quantilsfunktion eine einfache Darstellung in geschlossener Form. Durch die flexible Nachbildung unterschiedlicher Schiefe- und Kurtosiskonstellationen können beide Familien z.B. bei der Modellierung von Finanzmarktrenditen gewinnbringend eingesetzt werden.

Keywords: Hyperbolic secant; sinh-arcsinh Transformation, Skewness, Leptokurtosis, GARCH.

\section{Introduction}

GARCH models and generalizations are nowadays widely used for modeling financial returns and thus serve as models for the derivation of risk measures such as the Value-atRisk. The conditional distribution of such models are regularly found to be skewed and leptokurtic.

In order to model these findings a vast supply of parametric skewed and leptokurtic distributions has been proposed in recent years. Prominent examples are generalized t-distributions, with the latest example probably given in Zhu and Galbraith (2010), asymmetric power distributions, compare Komunjer (2007), stable distributions, compare e.g. Nolan (2010), or the very recent propositions of generalized maximum entropy distributions, see Herrmann (2011). All of these models are suitable for practical applications where the focus often is on Monte Carlo simulations or the derivation of the Value at Risk as a quantile of the loss distribution. For both applications it appears as a disadvantage that none of the mentioned distributions allows the derivation of a closed-form solution of the quantile function.

We derive two new distributions based on Jones and Pewsey (2009)'s sinh-arcsinh transformation of the hyperbolic secant distribution and Vaughan (2002)'s generalized 
secant hyperbolic distribution. These distributions exhibit similar flexibility for modeling skewness and kurtosis of financial data. In contrast to the above mentioned suggestions, both density, cumulative distribution and quantile function can be given in explicit form.

The outline of this article is as follows: We briefly discuss the sinh-arcsinh transformation in the first section. The next two sections introduce the two new distributions and discuss their properties. Section 4 compares their flexibility to the well-known financial models in an application to BMW returns. A conclusion is given in Section 5.

\section{The sinh-arcsinh Transformation}

Jones and Pewsey (2009) introduce the sinh-arcsinh transformation, briefly S-transformation, for some (symmetric) random variable $Z$ with known cumulative probability function $F_{Z}$ defined on $\mathbb{R}$. Let the $\mathrm{S}$-transformed random variable $X$ be given by

$$
Z=S(X ; \epsilon, \delta)=\sinh \left(\delta \sinh ^{-1}(X)-\epsilon\right),
$$

with $\epsilon \in \mathbb{R}$ and $\delta>0$. It follows from the monotonicity of $S$ that the distribution of $X$ depends on $Z$ 's and parameters $\epsilon, \delta$ with probability function given as

$$
F_{X}(x)=\operatorname{Pr}(X<x)=\operatorname{Pr}(S(X ; \epsilon, \delta)<S(x ; \epsilon, \delta))=F_{Z}(S(X ; \epsilon, \delta)) .
$$

Jones and Pewsey (2009) show that the parameters $\epsilon$ and $\delta$ of the transformed random variable $X$ act respectively as skewness and kurtosis parameters in the sense of the orderings defined in van Zwet (1964). Jones and Pewsey (2009) apply the sinh-arcsinhtransformation to introduce skewness and kurtosis to a normal distributed random variable. Rosco, Jones, and Pewsey (2010) apply this transformation to introduce skewness to a t-distributed random variable, where $\delta$ is hold constant at 1 . The following two sections discuss two further application of the sinh-arcsinh-transformation and discuss their results. The first approach introduces skewness and kurtosis to a hyperbolic secant distributed random variable. The second approach introduces skewness to an already leptokurtic or platykurtic generalized hyperbolic distributed random variable.

\section{Hyperbolic Secant Distribution}

For a hyperbolic secant distributed random variable $Z$, density function, cumulative probability function and quantile function are known in their explicit formulae as

$$
\begin{aligned}
f_{H S}(z) & =\frac{1}{2} \operatorname{sech}\left(\frac{\pi}{2} z\right), \\
F_{H S}(z) & =\frac{2}{\pi} \arctan \left(\exp \left(\frac{\pi}{2} x\right)\right), \\
F_{H S}^{-1}(u) & =\frac{2}{\pi} \log \left(\tan \left(\frac{\pi}{2} u\right)\right),
\end{aligned}
$$

where sech denotes the hyperbolic secant function. The distribution has zero mean and unit variance. It is symmetric and has an excess kurtosis of 2. 
Its elegant mathematical form already gave rise to further generalizations, e.g. in Harkness and Harkness (1968) or Vaughan (2002), see Section 5. Both generalizations define families of symmetric distributions with flexibility in terms of kurtosis. Contrary to these generalizations the S-transformation allows for both: introduction of skewness and kurtosis, see following section. Other generalizations of the hyperbolic distribution to skewed densities are given in Fischer (2004), Fischer (2006) and Fischer and Vaughan (2010). A recent overview is given in Fischer (2011).

\section{The HS-SAS Distribution}

The hyperbolic secant distribution is generalized to a skewed and leptokurtic distribution by the application of the S-transformation defined in (2) to the hyperbolic secant distribution defined in (3). The resulting S-transformed hyperbolic secant distribution, briefly HS-SAS distribution, is given by its probability function as

$$
F_{H S-S A S}(x ; \epsilon, \delta)=F_{H S}(S(x ; \epsilon, \delta))=\frac{2}{\pi} \arctan \left(\exp \left(\sinh \left(\delta \sinh ^{-1}(x)-\epsilon\right)\right)\right) .
$$

Its inverse defines the quantile function with the $u$-percentile $x_{u}$ as

$$
x_{u}=F_{H S-S A S}^{-1}(u ; \epsilon, \delta)=\sinh \left(\frac{\epsilon+\operatorname{arcsinh}(\log (\tan (1 / 2 u \pi)))}{\delta}\right) .
$$

The median thus only depends on $\epsilon$ and $\delta$ and is given by

$$
x_{0.5}=\sinh \left(\frac{\epsilon}{\delta}\right) .
$$

The density function is derived as the derivative of the probability function with respect to $x$ as

$$
f_{H S-S A S}(x ; \epsilon, \delta)=2 \frac{\cosh \left(-\delta \sinh ^{-1}(x)-\epsilon\right) \delta e^{-\sinh \left(-\delta \sinh ^{-1}(x)-\epsilon\right)}}{\pi \sqrt{1+x^{2}}\left(1+\left(e^{-\sinh \left(-\delta \sinh ^{-1}(x)-\epsilon\right)}\right)^{2}\right)} .
$$

The fact, shown in Jones and Pewsey (2009), that $\epsilon$ and $\delta$ act as skewness and kurtosis parameters respectively is illustrated in Figure 1.

Figure 2 illustrates the dependence of skewness and kurtosis on corresponding parameters $\epsilon$ and $\delta$. Skewness and kurtosis is measured as standardized third and fourth power moments (denoted as $m_{3}$ and $m_{4}$ ) as

$$
m_{3}=\mathrm{E}\left(\left(\frac{x-\mu}{\sigma}\right)^{3}\right), \quad m_{4}=\mathrm{E}\left(\left(\frac{x-\mu}{\sigma}\right)^{4}\right)
$$

and by use of two quantile based measures suggested in Bowley (1920) and Crow and Siddiqui (1967) (denoted as $q_{3}$ and $m_{4}$ ) as

$$
q_{3}=\frac{x_{0.75}+x_{0.25}-2 x_{0.5}}{x_{0.75}-x_{0.25}}
$$



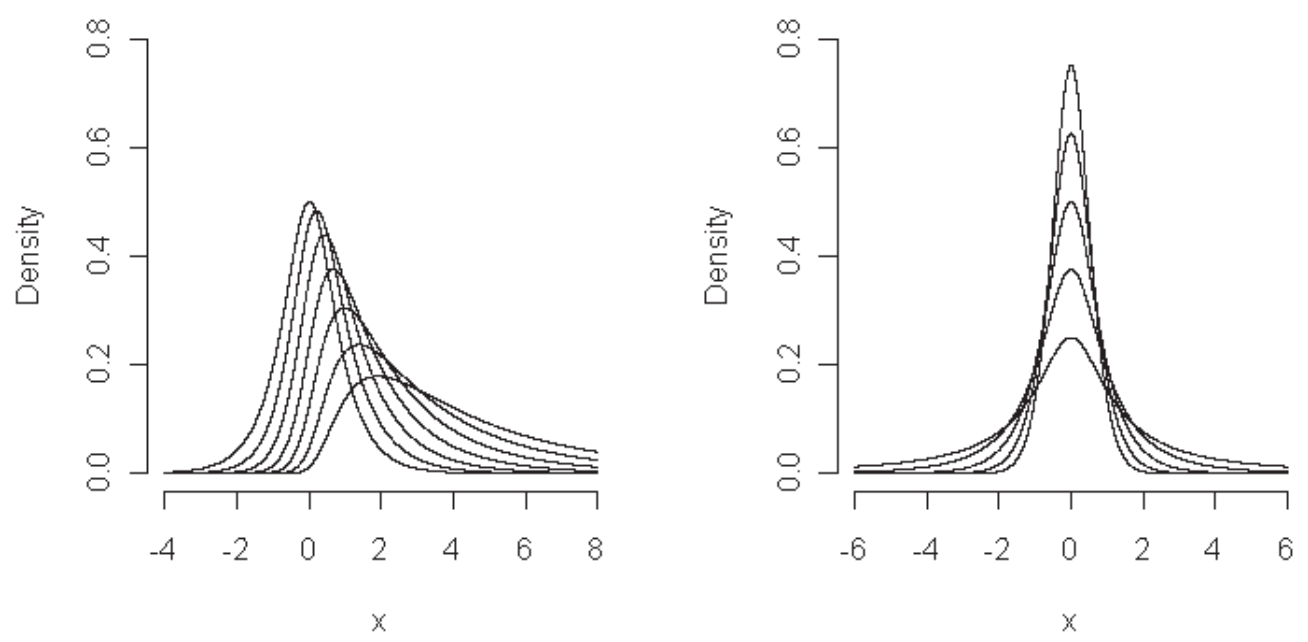

Figure 1: The S-transformed hyperbolic secant distribution with $\epsilon \in[0 ; 2], \delta=1$ (left panel), and $\epsilon=0, \delta \in[0.5 ; 1.5]$ (right panel).

and

$$
q_{4}=\frac{x_{0.975}-x_{0.025}}{x_{0.75}-x_{0.25}},
$$

where $x_{u}$ denotes the quantile belonging to probability $u$. While standardized power moments are calculated via numerical integration, the quantile based measures can be derived directly from the quantile function.

Finally, we will demonstrate that all moments of an HS-SAS distribution exist: Firstly, the $n$-th power of the $\mathrm{S}$-transformation derives as

$$
\begin{aligned}
S(z)^{n} & =\frac{1}{2^{n}} \sum_{i=0}^{n}\left(\begin{array}{l}
n \\
i
\end{array}\right) e^{\epsilon(n-2 i)}\left(z+\sqrt{z^{2}+1}\right)^{\delta(n-2 i)} \\
& =\frac{1}{2^{n}} \sum_{i=0}^{n}\left(\begin{array}{l}
n \\
i
\end{array}\right) e^{\epsilon(n-2 i)} \exp \left\{\delta(n-2 i) \log \left(\left(z+\sqrt{z^{2}+1}\right)\right)\right\} .
\end{aligned}
$$

Replacing $z$ by $X$ and taking expectations, we obtain

$$
\begin{aligned}
\mathrm{E}\left(Z^{n}\right) & =\frac{1}{2^{n}} \sum_{i=0}^{n}\left(\begin{array}{c}
n \\
i
\end{array}\right) e^{\epsilon(n-2 i)} \mathrm{E}(\exp (\delta(n-2 i) W)) \\
& =\frac{1}{2^{n}} \sum_{i=0}^{n}\left(\begin{array}{c}
n \\
i
\end{array}\right) e^{\epsilon(n-2 i)} \mathcal{M}_{W}(\delta(n-2 i))
\end{aligned}
$$

with $W \equiv \operatorname{asinh}(Z)=\log \left(Z+\sqrt{Z^{2}+1}\right)$ and where $\mathcal{M}_{W}$ denotes the corresponding moment-generating function. Some properties of $W$ are the following: Assume that $Z$ 
M3 of HS-SAS for $\delta=1$

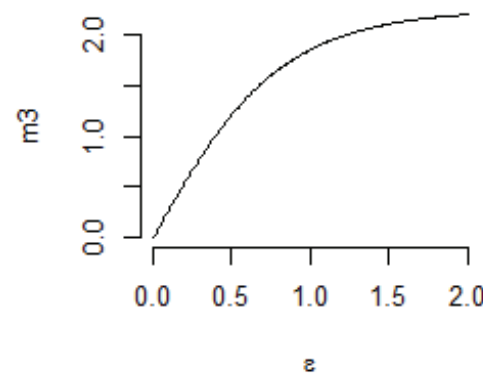

M4 of HS-SAS for $\varepsilon=0$

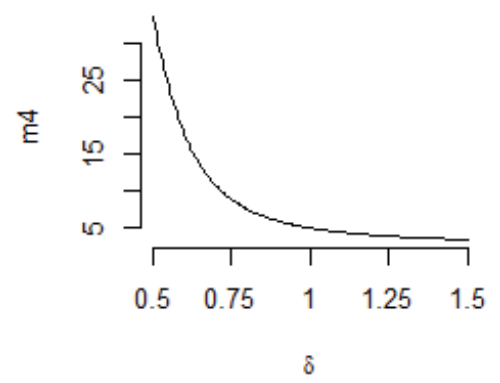

Q3 of HS-SAS for $\delta=1$

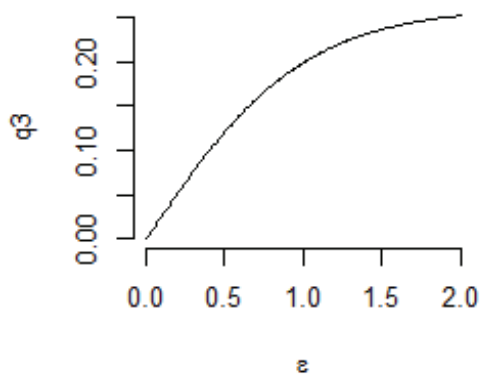

Q4 of HS-SAS for $\varepsilon=0$

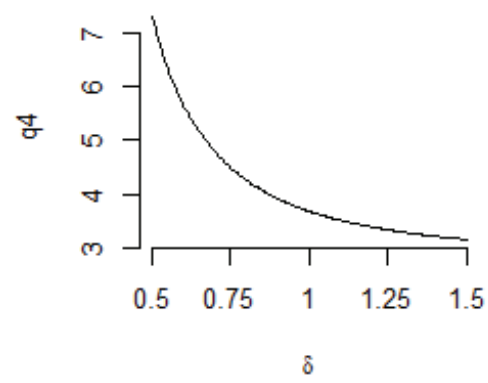

Figure 2: Moment statistics and quantile measures for the S-transformed hyperbolic secant distribution varying with $\epsilon$ and $\delta$.

represents the hyperbolic secant density. The underlying transformation $\mathrm{T}$ is monotone increasing because

$$
\mathrm{T}^{\prime}(x)=\frac{1}{\sqrt{x^{2}+1}}>0 .
$$

Because of its inverse function, $\mathrm{T}^{-1}(x)=\sinh (x)$ with corresponding first derivative $\left(\mathrm{T}^{-1}\right)^{\prime}(x)=\cosh (x)>0$, the random variable $W$ admits the following density (see Figure 3)

$$
f_{W}(x)=\frac{\cosh (x)}{\pi \cosh (\sinh (x))}, \quad x \in \mathbb{R}
$$

with cumulative distribution function

$$
F_{W}(x)=\frac{2}{\pi} \arctan (\exp (\cosh (x))), \quad x \in \mathbb{R} .
$$

It has light tails (such that all moments exist). The even ones can be numerically approximated as

$$
\begin{aligned}
& \mathrm{E}(W)=0, \quad \mathrm{E}\left(W^{2}\right)=1.0655, \quad \mathrm{E}\left(W^{3}\right)=0, \quad \mathrm{E}\left(W^{4}\right)=2.587 \\
& \mathrm{E}\left(W^{6}\right)=8.813, \quad \mathrm{E}\left(W^{8}\right)=37.009 .
\end{aligned}
$$



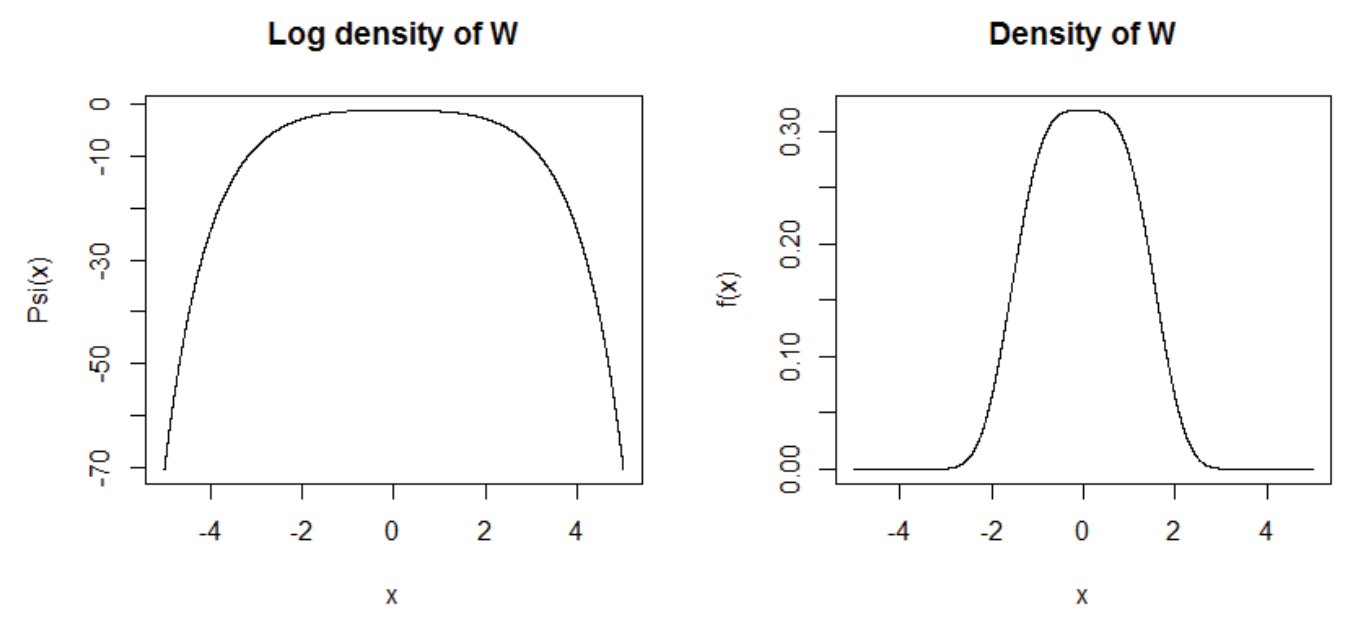

Figure 3: Log density (left panel) and density (right panel) of $W$.

\section{Skewing Vaughan's GSH distribution}

Vaughan (2002) suggests the following generalization of the hyperbolic distribution,

$$
f_{G S H}(x ; t)=c_{1} \frac{\exp \left(c_{2} x\right)}{\exp \left(2 c_{2} x\right)+2 a \exp \left(c_{2} x\right)+1}
$$

for $t>-\pi$, where two cases for $t$ are distinguished as

$$
\begin{array}{ll}
t \leq 0: & a(t)=\cos (t), \quad c_{1}(t)=\frac{\sin (t)}{t} c_{2}, \quad c_{2}(t)=\sqrt{\frac{\pi^{2}-t^{2}}{3}} \\
t>0: & a(t)=\cosh (t), \quad c_{1}(t)=\frac{\sinh (t)}{t} c_{2}, \quad c_{2}(t)=\sqrt{\frac{\pi^{2}+t^{2}}{3}}
\end{array}
$$

The parameter $t$ drives the distribution's shape in terms of kurtosis but leaves the distribution symmetric, see Vaughan (2002). Distribution and quantile function are also known as

for $\pi<t<0$ :

$$
\begin{aligned}
& F_{G S H}(x ; t)=1+\frac{1}{t} \cot ^{-1}\left(\frac{\exp \left(c_{2} x\right)+\cos (t)}{\sin (t)}\right), \\
& F_{G S H}^{-1}(u ; t)=\frac{1}{c_{2}} \log \left(\frac{\sin (t u)}{\sin (t(1-u))}\right),
\end{aligned}
$$

with $a(t)=\cos (t), c_{1}(t)=\frac{\sin (t)}{t} c_{2}, c_{2}(t)=\sqrt{\frac{\pi^{2}-t^{2}}{3}}$; 
for $t=0$ :

$$
\begin{aligned}
& F_{G S H}(x ; 0)=\exp \left(\frac{\pi x}{\sqrt{3}}\right)\left(1+\exp \left(\frac{\pi x}{\sqrt{3}}\right)\right)^{-1}, \\
& F_{G S H}^{-1}(x ; 0)=\frac{\sqrt{3}}{\pi} \log \left(\frac{u}{1-u}\right),
\end{aligned}
$$

with $a(t)=1, c_{1}(t)=c_{2}, c_{2}(t)=\frac{\pi}{\sqrt{3}}$;

for $t>0$ :

$$
\begin{aligned}
& F_{G S H}(x ; t)=1-\frac{1}{t} \operatorname{coth}^{-1}\left(\frac{\exp \left(c_{2} x\right)+\cosh (t)}{\sinh (t)}\right), \\
& F_{G S H}^{-1}(x ; t)=\frac{1}{c_{2}} \log \left(\frac{\sinh (t u)}{\sinh (t(1-u))}\right),
\end{aligned}
$$

with $a(t)=\cosh (t), c_{1}(t)=\frac{\sinh (t)}{t} c_{2}, c_{2}(t)=\sqrt{\frac{\pi^{2}+t^{2}}{3}}$.

As the GSH distribution already possesses flexibility towards kurtosis the S-transformation is applied only to introduce skewness. This is done by applying the S-transformation with constant $\delta=1$ and variable $\epsilon \in \mathbb{R}$ (similar to Rosco et al., 2010 for the Student$\mathrm{t}$ distribution). The S-skewed GSH distribution, briefly GSH-SAS, is thus given by the S-transformed random variable $X$ with probability function

$$
F_{G S H-S A S}(x ; \epsilon, t)=F_{G S H}(S(x ; \epsilon, 1) ; t) .
$$

Probability, probability density and quantile function can be given in explicit form, where it must again be distinguished for $t$ as

for $-\pi<t<0$ :

$$
\begin{gathered}
F_{G S H-S A S}(x ; \epsilon, t)=1+\left(\pi-\operatorname{arccot}\left(\frac{e^{-c \sinh (\epsilon-\operatorname{arcsinh}(x))}+\cos (t)}{\sin (t)}\right)\right) t^{-1} \\
f_{G S H-S A S}(x ; \epsilon, t)=\frac{c \cosh (\epsilon-\arcsin (x)) e^{-c \sinh (\epsilon-\operatorname{arcsinh}(x))}}{t \sqrt{1+x^{2}} \sin (t)\left(1+e^{\left.-c \sinh (\epsilon-\operatorname{arcsinh}(x))+\cos (t)^{2} \sin (t)^{-2}\right)}\right.} \\
F_{G S H-S A S}^{-1}(u ; \epsilon, t)=\sinh \left(\epsilon+\operatorname{arcsinh}\left(\frac{\log (-\cos (t)-\cot (-t+u t) \sin (t))}{c}\right)\right)
\end{gathered}
$$

with $c(t)=\sqrt{\frac{\pi^{2}-t^{2}}{3}}$;

for $t=0$ :

$$
\begin{gathered}
F_{G S H-S A S}(x ; \epsilon, 0)=\frac{\gamma(x)}{1+\gamma(x)} \\
f_{G S H-S A S}(x ; \epsilon, 0)=\sqrt{\frac{\pi}{3}} \frac{\gamma(x)}{\sqrt{1+x^{2}}} \cosh (\epsilon-\operatorname{arcsinh}(x))\left(\frac{1}{1+\gamma(x)}-\frac{\gamma(x)}{(1+\gamma(x))^{2}}\right) \\
F_{G S H-S A S}^{-1}(u ; \epsilon, 0)=\sinh \left(\epsilon+\operatorname{arcsinh}\left(\frac{\sqrt{3}}{\pi} \log \left(\frac{u}{1-u}\right)\right)\right)
\end{gathered}
$$


with $\gamma(x)=\exp \left(-\sqrt{\frac{1}{3}} \pi \sinh (\epsilon-\operatorname{arcsinh}(x))\right)$,

for $t>0$ :

$$
\begin{gathered}
F_{G S H-S A S}(x ; \epsilon, t)=1-\frac{1}{t} \operatorname{arccoth}\left(\frac{e^{-c \sinh (\epsilon-\operatorname{arcsinh}(x))}+\cosh (t)}{\sinh (t)}\right) \\
f_{G S H-S A S}(x ; \epsilon, t)=\frac{c \cosh (\epsilon-\operatorname{arcsinh}(x)) e^{-c \sinh (\epsilon-\operatorname{arcsinh}(x))}}{t \sqrt{1+x^{2}} \sinh (t)\left(\left(e^{\left.-c \sinh (\epsilon-\operatorname{arcsinh}(x))+\cosh (t))^{2} \sinh (t)^{-2}-1\right)}\right.\right.} \\
F_{G S H-S A S}^{-1}(u ; \epsilon, t)=\sinh \left(\epsilon+\operatorname{arcsinh}\left(\frac{\log (-\cosh (t)-\operatorname{coth}(-t+u t) \sinh (t))}{c}\right)\right) \\
\text { with } c(t)=\sqrt{\frac{\pi^{2}+t^{2}}{3}} .
\end{gathered}
$$

The new distribution's flexibility in terms of skewness is illustrated in Figure 4.
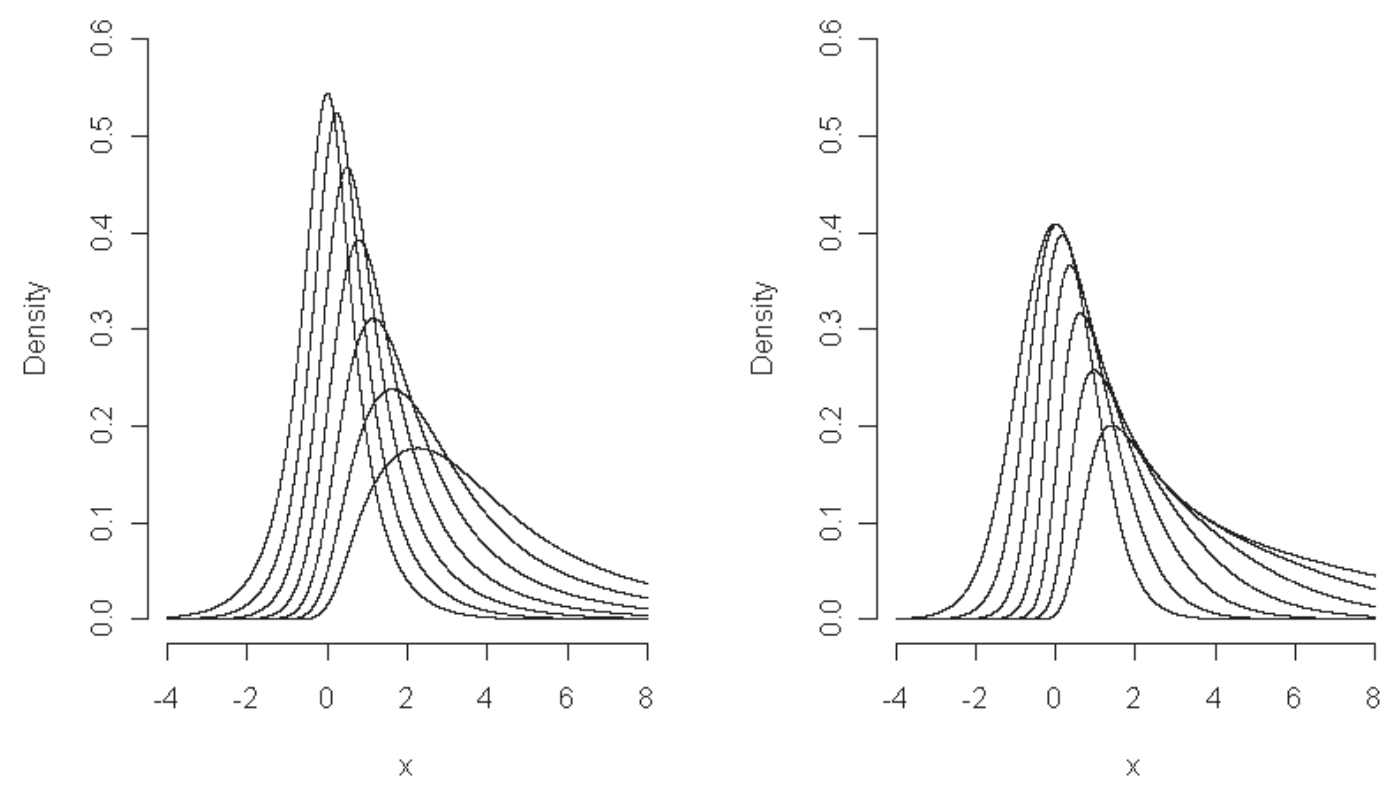

Figure 4: The skewed generalized hyperbolic secant distribution with $\epsilon \in[0 ; 2], \delta=-2$ (left panel), and $\delta=2$ (right panel).

The effect of the skewness parameter on measures of skewness is illustrated in Figure 5.

\section{Application to Univariate Returns}

We apply the new models to daily BMW share prices and their log-returns from January 1st 2009 to March 15th 2011, see Figure 6. As Figure 6 indicates there could be GARCHtype effects in return volatility such that we consider both standardized and GARCHfiltered returns, see Table $1 .^{1}$ It can be seen that filtering for GARCH-effects reduces both

\footnotetext{
${ }^{1}$ The GARCH-filter is based on a GARCH$(1,1)$ process.
} 

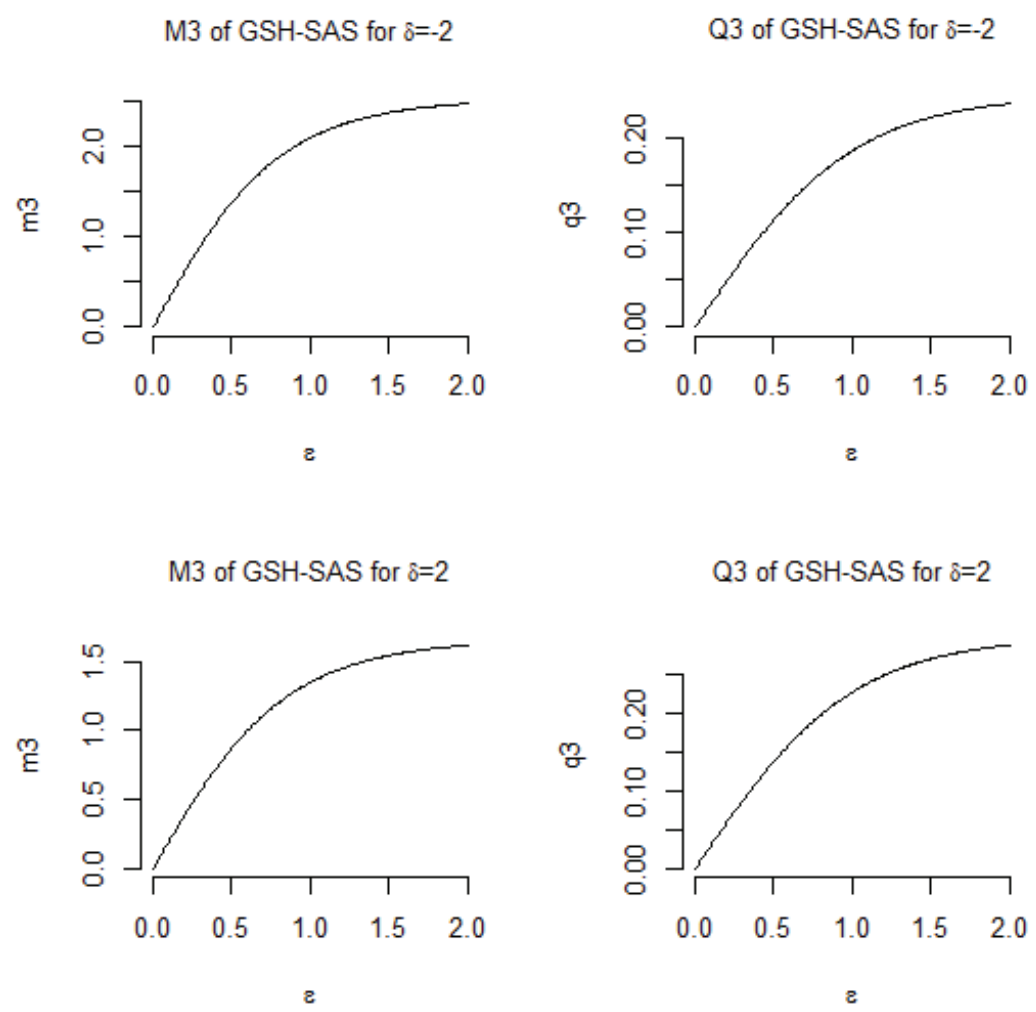

Figure 5: Two measures of skewness for the S-skewed generalized hyperbolic secant distribution varying with $\epsilon$.

skewness and kurtosis, both measured by third or fourth standardized power moments, respectively.

Both, pronounced kurtosis and positive skewness, indicate a significant deviation from normality in both types of returns. We thus apply both the S-transformed hyperbolic secant distribution and the skewed generalized hyperbolic distribution to fit the returns. A skewed t-distribution derived from the maximum entropy framework proposed in Herrmann (2011) denoted as MEHC and the normal distribution serve as benchmark for both models. For the former we choose a arctan-based moment to measure skewness as in Fischer, Gao, and Herrmann (2010). This density function has been found to have similar flexibility for modeling financial market returns as other skewed t-distributions, e.g. the SGT2 proposed in Grottke (2001), compare Fischer et al. (2010). As skewness in both, raw and GARCH-filtered data, is less pronounced than kurtosis, a symmetrical and the skewed version is estimated for each of the suggested distributions.

In order to assess the different models flexibility we apply different goodness-offit criteria such as likelihood $(\mathcal{L L})$ and the likelihood-based information Akaike and Bayesian information criterion $(\mathcal{A I C}$ and $\mathcal{B I C})$. Together with the Kolmogorov-Smirnov $(K S)$ and $\chi^{2}$ distance, these measures emphasize the overall fit. The last criterion chosen is the Andersen-Darling distance $(\mathcal{A D})$ that puts more emphasis on the fit in the tails. 
Table 1: Descriptive statistics for the data used.

\begin{tabular}{l|c|c} 
& Raw returns & GARCH-filtered returns \\
\hline Mean & 0.0016 & 0.0712 \\
Standard Deviation & 0.0242 & 1.0027 \\
Skewness & 0.4218 & 0.2946 \\
Kurtosis & 6.0083 & 4.1232 \\
Observations & 561 & 561
\end{tabular}

BMW Share Price

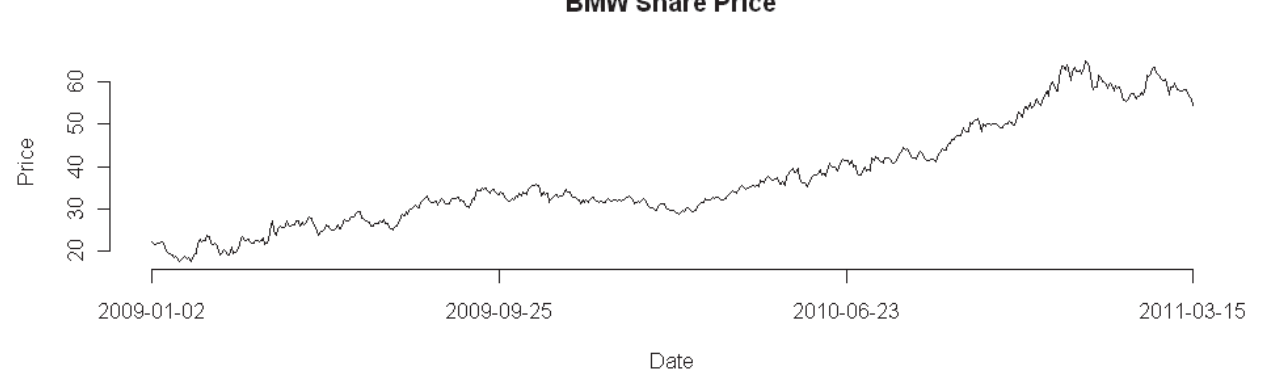

BMW Log-Return

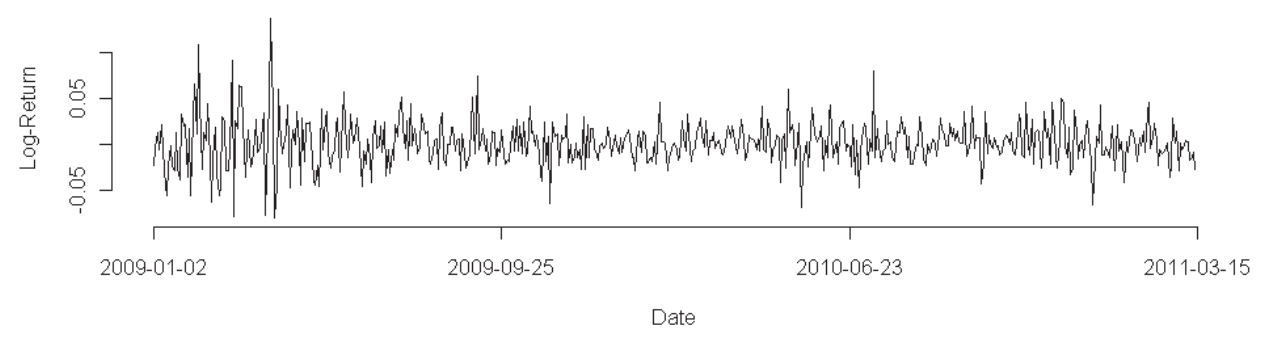

Figure 6: BMW share price and its log-return.

A graphical representation of the fitted distribution is given in Figure $7 .^{2}$ Results for goodness-of-criteria are given in Tables 3 and 5, estimates in Tables 2 and 4.

The skewness parameters for all distributions are significantly different from 0 (the value implying symmetry) and the skewed version of every distribution outperforms the symmetric versions by all criteria of goodness-of-fit. The new distributions outperform the normal distribution by all criteria including those which penalize additional parameters $(\mathcal{A I C}$ and $\mathcal{B I C})$. For the case of raw returns the skewed t-distribution is slightly better as the new suggestions. But for the GARCH-filtered returns there is mixed picture: The t-distribution gives a lower $\chi^{2}$ distance, but worse $K S$ distance and likelihood-based criteria. Both new suggestions give a marginal better fit in the tails for GARCH-filtered returns but worse fit for raw returns.

\footnotetext{
${ }^{2}$ For HS-SAS, GSH-SAS and MEHC only the skewed version is plotted.
} 

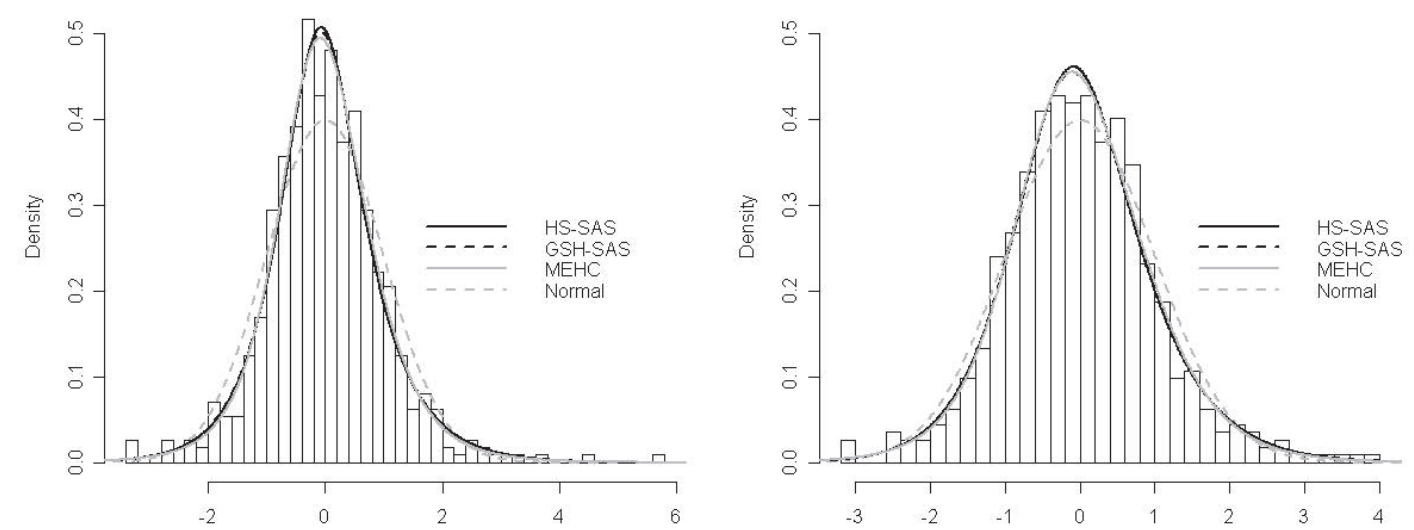

Figure 7: Histogramm and fitted density functions for BMW share price and its log-return.

Table 2: Estimated parameters and standard deviations for BMW raw returns.

\begin{tabular}{l|c|c|c|c|c|} 
Distribution & $\delta$ & $t$ & $\epsilon$ & $\nu$ & $\arctan ^{2}$ \\
\hline HS-SAS (sym.) & 0.98016 & - & - & - & - \\
\multirow{2}{*}{ HS-SAS (skw.) } & 0.00561 & - & - & - & - \\
\multirow{3}{*}{ GSH } & 0.97990 & - & 0.09229 & - & - \\
& 0.00571 & - & 0.00450 & - & - \\
GSH-SAS & - & -1.56043 & - & - & - \\
\multirow{2}{*}{ MEHC (sym.) } & - & 0.11467 & - & - & - \\
\multirow{2}{*}{ MEHC (skw.) } & - & -1.57346 & 0.09477 & - & - \\
& - & 0.11165 & 0.00468 & - & - \\
& - & - & - & 2.93645 & - \\
& - & - & - & 0.00202 & - \\
& & - & - & 2.93645 & -0.00780 \\
& & & & 0.00202 & $4.47 \mathrm{e}-05$
\end{tabular}

Table 3: Goodness-of-fit for BMW raw returns.

\begin{tabular}{l|c|c|c|c|c|c} 
Distribution & $\mathcal{L L}$ & $\mathcal{A I C}$ & $\mathcal{B I C}$ & $K S$ & $\chi^{2}$ & $\mathcal{A D}$ \\
\hline HS-SAS (sym.) & -771.7 & 1551.4 & 1568.8 & 3.14 & 6.485 & 0.160 \\
HS-SAS (skw.) & -770.8 & 1549.5 & 1566.9 & 1.79 & 4.842 & 0.118 \\
GSH & -771.8 & 1551.5 & 1568.8 & 3.01 & 6.402 & 0.176 \\
GSH-SAS & -770.8 & 1549.6 & 1566.9 & 1.87 & 4.789 & 0.125 \\
MEHC (sym.) & -770.6 & 1549.2 & 1566.5 & 2.87 & 5.119 & 0.070 \\
MEHC (skw.) & -769.9 & $\mathbf{1 5 4 7 . 9}$ & $\mathbf{1 5 6 5 . 2}$ & $\mathbf{1 . 6 9}$ & $\mathbf{3 . 3 5 4}$ & $\mathbf{0 . 0 6 4}$ \\
Normal & -795.5 & 1595 & 1603.7 & 4.61 & 21.603 & 18.22
\end{tabular}


Table 4: Estimated parameters and standard deviations for BMW GARCH-filtered returns.

\begin{tabular}{|c|c|c|c|c|c|}
\hline Distribution & $\delta$ & $t$ & $\epsilon$ & $\nu$ & $\arctan ^{2}$ \\
\hline \multirow[t]{2}{*}{ HS-SAS (sym.) } & 1.18057 & - & - & - & - \\
\hline & 0.01442 & - & - & - & - \\
\hline \multirow[t]{2}{*}{ HS-SAS (skw.) } & 1.19732 & - & 0.15360 & - & - \\
\hline & 0.01569 & - & 0.00886 & - & - \\
\hline \multirow[t]{2}{*}{ GSH } & - & 0.03329 & - & - & - \\
\hline & - & 60.8726 & - & - & - \\
\hline \multirow[t]{2}{*}{ GSH-SAS } & - & 0.25462 & 0.10926 & - & - \\
\hline & - & 8.27093 & 0.00454 & - & - \\
\hline \multirow[t]{2}{*}{ MEHC (sym.) } & - & - & - & 4.0960 & - \\
\hline & - & - & - & 0.00290 & - \\
\hline \multirow[t]{2}{*}{ MEHC (skw.) } & - & - & - & 4.2280 & -0.00780 \\
\hline & - & - & - & 0.00306 & $4.44 \mathrm{e}-05$ \\
\hline
\end{tabular}

Table 5: Goodness-of-fit for BMW GARCH-filtered returns.

\begin{tabular}{l|c|c|c|c|c|c} 
Distribution & $\mathcal{L L}$ & $\mathcal{A I C}$ & $\mathcal{B I C}$ & $K S$ & $\chi^{2}$ & $\mathcal{A D}$ \\
\hline HS-SAS (sym.) & -786.7 & 1581.5 & 1598.8 & 2.64 & 6.475 & 0.107 \\
HS-SAS (skw.) & -785.3 & 1578.6 & 1595.9 & $\mathbf{1 . 7 4}$ & 5.311 & 0.069 \\
GSH & -786.4 & 1580.9 & 1598.2 & 2.60 & 5.479 & 0.102 \\
GSH-SAS & -785.1 & $\mathbf{1 5 7 8 . 3}$ & $\mathbf{1 5 9 5 . 6}$ & 1.75 & 4.399 & $\mathbf{0 . 0 6 7}$ \\
MEHC (sym.) & -786.5 & 1581.1 & 1598.4 & 2.60 & 4.816 & 0.088 \\
MEHC (skw.) & -785.7 & 1579.4 & 1596.7 & 1.84 & $\mathbf{3 . 9 6 9}$ & 0.074 \\
Normal & -795.5 & 1595 & 1603.7 & 4.07 & 10.824 & 0.337
\end{tabular}

\section{Conclusion}

The sinh-arcsinh transformation introduced in Jones and Pewsey (2009) provides a useful tool for deriving new skewed and leptokurtic distributions. The application of such transformations to the hyperbolic secant and the generalized hyperbolic distributions yields new flexible distributions for which all, probability, probability density and quantile function can be given in closed-form. The exemplary empirical study shows that the flexibility of such distributions is not necessarily outperformed by other flexible distributions typically applied for financial market models.

\section{References}

Bowley, A. L. (1920). Elements of Statistics. New York: Charles Scribner's Sons.

Crow, E. L., and Siddiqui, M. M. (1967). Robust estimation of location. Journal of the American Statistical Association, 62, 353-389. 
Fischer, M. (2004). Skew generalized secant hyperbolic distributions: Unconditional and conditional fit to asset returns. Austrian Journal of Statistics, 33, 293-304.

Fischer, M. (2006). The skew generalized secant hyperbolic family. Austrian Journal of Statistics, 35, 437-443.

Fischer, M. (2011). Hyperbolic secant distributions and generalizations. In M. Lovric (Ed.), International Encyclopedia of Statistical Sciences. Springer.

Fischer, M., Gao, Y., and Herrmann, K. (2010). Volatility models with innovations from new maximum entropy densities at work. IWQW Discussion Paper Series, 03.

Fischer, M., and Vaughan, D. (2010). The beta-hyperbolic secant distribution. Austrian Journal of Statistics, 39, 245-258.

Grottke, M. (2001). Die t-Verteilung und ihre Verallgemeinerungen als Modell für Finanzmarktdaten. Köln.

Harkness, W. L., and Harkness, M. L. (1968). Generalized hyperbolic secant distributions. Journal of the American Statistical Association, 63, 329-337.

Herrmann, K. (2011). Maximum Entropy Models for Time-Varying Moments Applied to Daily Financial Returns. Unpublished doctoral dissertation, University of Erlangen Nürnberg.

Jones, M. C., and Pewsey, A. (2009). Sinh-arcsin distributions. Biometrika, 96, 761-780.

Komunjer, I. (2007). Asymmetric power distribution: Theory and applications to risk management. Journal of Applied Econometrics, 22, 821-921.

Nolan, J. P. (2010). Stable distributions - models for heavy tailed data. (To be published)

Rosco, J. F., Jones, M. C., and Pewsey, A. (2010). Skew t distributions via the sinh-arcsinh transformation. Test. (10.1007/s11749-010-0222-2)

van Zwet, W. R. (1964). Convex Transformations of Random Variables. Unpublished doctoral dissertation, Amsterdam University.

Vaughan, D. C. (2002). The generalized secant hyperbolic distribution and its properties. Communications in Statististics - Theory and Method, 31, 219-238.

Zhu, D., and Galbraith, J. W. (2010). A generalized asymmetric Student t-distribution with application to financial econometrics. Journal of Econometrics, 157, 297-305.

Authors' address:

Matthias Fischer and Klaus Herrmann

Universität Erlangen-Nürnberg

Lehrstuhl für Statistik und Ökonometrie

Lange Gasse 20

D-90403 Nürnberg

E-mail: Matthias.Fischer@wiso.uni-erlangen.de, Klaus_Herrmann@McKinsey.com 